\title{
Morbidity from asthma in relation to regular treatment: a community based study
}

\author{
L J Walsh, C A Wong, S Cooper, A R Guhan, M Pringle, A E Tattersfield
}

\begin{abstract}
Background-The extent to which asthma morbidity in the community occurs in patients who are having relatively little treatment or in those on step 3 or above of the British asthma management guidelines is uncertain. We have looked at this in a community population in southern Nottinghamshire.

Methods-A cross sectional review of treatment in all patients over the age of four with diagnosed asthma was carried out in five large general practices (population 38 865) in 1995/6 using computerised general practice records. The patients' usual treatment was obtained from prescription data and categorised by the appropriate step on the British guidelines on asthma management. Two measures of morbidity, the request for 10 or more short acting $\beta$ agonist inhalers a year or the need for a course of oral corticosteroids in the last year, were related to the regular treatment of the patients.

Results-Of the 3373 patients $(8.7 \%)$ given a diagnosis of asthma, the percentage on steps $1,2,3,4$, and 5 of treatment were $54 \%, 22 \%, 11 \%, 3.6 \%$, and $1 \%$, respectively, with a further $8 \%$ having had no treatment. During the past year $13.6 \%$ had been prescribed 10 or more $\beta$ agonist inhalers and $12.5 \%$ had received at least one course of oral corticosteroids. Both measures occurred more frequently in patients taking more prophylactic treatment (step 3 or above). Nevertheless, because most patients were on steps 1 and 2 of the treatment guidelines, more than half the patients requiring high doses of inhaled $\beta$ agonists or a course of oral prednisolone came from those taking low dose or no regular inhaled corticosteroid. Conclusions-Evidence of morbidity from asthma was found in many patients taking little or no prophylactic medication and this should be amenable to improved education. A different approach may be needed for patients with continuing morbidity who are already taking higher doses of prophylactic medication.

(Thorax 1999;54:296-300)
\end{abstract}

Keywords: asthma; treatment; British Thoracic Society; guidelines; morbidity

Many patients with asthma continue to have frequent symptoms and exacerbations for which they take inhaled short acting $\beta$ agonists or a course of oral corticosteroids. The
Table 1 Outline of the management steps of the British guidelines for asthma management

$\begin{array}{ll}\text { Step 1 } & \begin{array}{l}\text { Bronchodilator only } \\ \text { Step 2 } \\ \text { or budesonide or } \leqslant 200 \mu \mathrm{g} \text { fluticasone) or regular } \\ \text { sodium cromoglycate or nedocromil sodium } \\ \text { High dose inhaled corticosteroid }(\geqslant 800 \mu \mathrm{g} / \text { day BDP } \\ \text { or budesonide, or } \geqslant 500 \mu \mathrm{g} \text { fluticasone) or low dose } \\ \text { (as for step 2) plus a long acting } \beta \text { agonist }\end{array} \\ \text { Step } & \begin{array}{l}\text { High dose inhaled corticosteroid (as for step 3) plus } \\ \text { a regular bronchodilator }\end{array} \\ \text { Segular oral corticosteroid therapy }\end{array}$

$\mathrm{BDP}=$ beclomethasone dipropionate.

From British Thoracic Society et al. ${ }^{1}$

frequent use of short acting $\beta$ agonists and the need for short courses of oral corticosteroids can be used as measures of poor asthma control in a community population. How these two measures relate to patients' usual treatment is uncertain but is important in determining strategies to reduce morbidity. Continuing symptoms and exacerbations in patients taking relatively little regular treatment may be preventable through education and more appropriate use of prophylactic medication and management plans. The occurrence of similar problems despite recommended doses of prophylactic medication suggests that the patient has more severe asthma and that a different approach is required.

A patient's regular treatment for asthma can be summarised in relation to the five steps on the current British asthma management guidelines ${ }^{1}$ which range from step 1 , reflecting occasional $\beta$ agonists, to step 5 which includes regular oral corticosteroids (table 1 ). We have used computerised data on drug prescriptions from general practices in Nottinghamshire to provide a community based profile of the proportion of patients with asthma on each step of the treatment guidelines. We then looked at how the use of high doses of short acting $\beta$ agonists and the need for oral corticosteroids relate to patients' regular treatment, as described by the step on the guidelines. These data provide information on the spectrum of disease in a community population as measured by the asthma treatment, and give some indication of the extent to which recurrent symptoms and exacerbations may or may not be preventable.

\section{Methods}

The survey took place in 1995/6 and covered a population of 38865 patients over the age of four in five large general practices in southern Nottinghamshire. The practices had computerised patient records that included details of diagnoses and all drugs prescribed for at least four years and were identified with the help of 
the Nottingham Family Health Service Authority (FHSA). ${ }^{2}$ The five practices were run by 24 general practitioners and included two rural practices, one practice from an inner city area, and two from suburban Nottingham. The study was approved by Nottingham City Hospital ethics committee and by Nottingham FHSA.

All patients over the age of four who were currently registered with the practice and had a diagnosis of asthma, with or without a diagnosis of chronic obstructive pulmonary disease (COPD), were identified from the practice computerised records. Each patient's computer record was reviewed to document patient details, major diagnoses, current asthma medication, and treatment over the previous four years. This included details of the type of inhaled corticosteroid, the dose and number of canisters prescribed and the period over which they were issued, and all courses of oral corticosteroid for asthma. Asthma medication included inhaled or oral bronchodilators, sodium cromoglycate, nedocromil sodium, and inhaled corticosteroids. The use of regular oral corticosteroids and the condition for which they were prescribed was recorded.

We used prescription prescribing, analysis and cost data (PACT) to compare prescribing in the five practices with that for Nottinghamshire for the year 1993/4. The number of prescriptions for prophylactic medications (inhaled steroids, sodium cromoglycate, and nedocromil sodium) and for all inhaled and oral bronchodilators was related to the total population in the practice and in Nottinghamshire to obtain the number of prescriptions per person.

Each patient with asthma was allocated to a step on the 1995 British guidelines on asthma management according to their current treatment regimen (table 1). Patients with a diagnosis of both asthma and COPD were included since the numbers were small and it was not possible to determine the main diagnosis from the computer record. Patients were described as being on regular inhaled

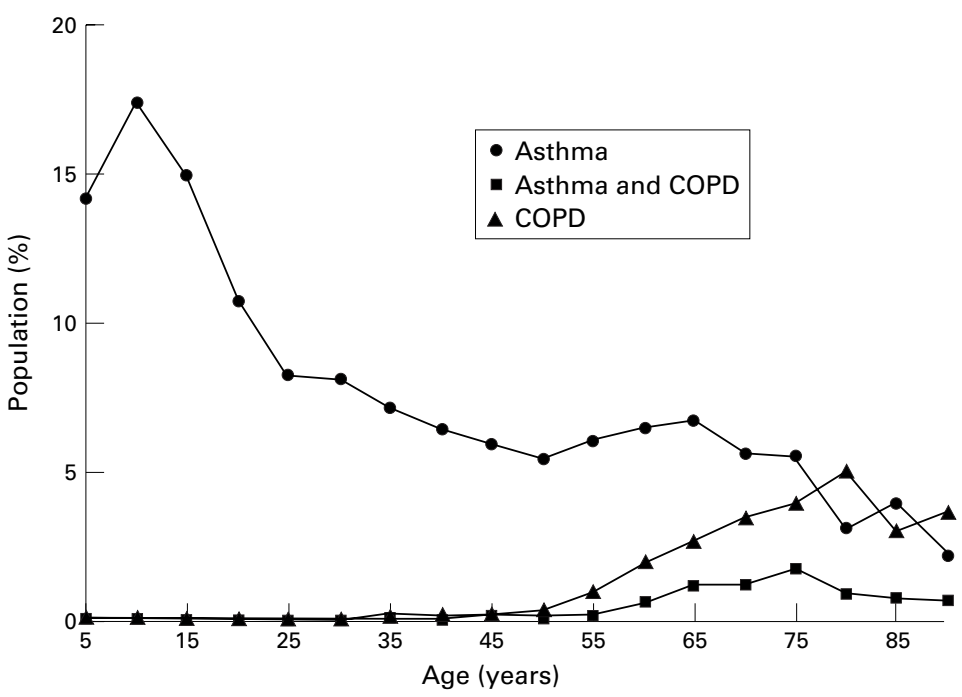

Figure 1 Prevalence of diagnosed asthma and chronic obstructive pulmonary disease (COPD) in a Nottinghamshire general practice population of 38865 patients. corticosteroids when they had requested sufficient corticosteroid inhalers to provide at least 600 doses a year since commencing treatment, and as having intermittent inhaled corticosteroids when they requested less. Patients were allocated to step 4 if they were taking a regular bronchodilator at the time of the survey and to step 5 if they had taken oral corticosteroids continuously for six months. The numbers of patients requiring a course of oral corticosteroids and high doses of inhaled $\beta$ agonists in the past year were related to each step of the guidelines as a percentage of the patients on each step and in absolute numbers using SPSS 7.5 for Windows (SPSS Inc, Chicago, Illinois, USA).

\section{Results}

\section{STUDY POPULATION}

The 38865 subjects in the five practices showed a similar age and sex distribution to the population of Nottinghamshire $(52 \%$ versus $51 \%$ female; $16.5 \%$ versus $15.4 \%$ over age 65 ). The practices were sited in areas with Townsend scores ranging from -4.5 at the affluent end of the scale to +11.6 at the deprived end compared with -7.7 to +11.6 in Nottinghamshire. ${ }^{3}$ Analysis of the PACT data suggests that prescribing in the five study practices is similar to that seen in Nottinghamshire as a whole. The mean number of prophylactic and bronchodilator prescriptions per person in the practice population was 0.19 and 0.4 , respectively, for the study practices compared with 0.2 and 0.45 for Nottinghamshire.

The analysis was carried out on 3373 patients $(8.7 \%)$ with a diagnosis of asthma of whom $109(3 \%)$ also had a diagnosis of COPD (fig 1).

\section{REGULAR TREATMENT}

The percentage of patients receiving no treatment and on steps $1,2,3,4$, and 5 of the 1995 British guidelines on asthma management were $8 \%, 54 \%, 22 \%, 11 \%, 3.6 \%$, and $1 \%$, respectively (fig 2 ). Although over half the patients were on step 1 , half of these had received some inhaled corticosteroids but did not fulfil our criteria for regular use (shown as step $1 \mathrm{~b}$, clear box).

A third of patients were prescribed a regular inhaled corticosteroid (table 2) and 33\% of these were prescribed $800 \mu \mathrm{g}$ or more per day. Most patients on step 3 were receiving high dose inhaled corticosteroids rather than a low dose inhaled corticosteroid and a long acting bronchodilator ( $84 \%$ versus $16 \%$ ).

Thirty five patients were taking regular oral corticosteroids for asthma of whom 34 were taking prednisolone (median dose $7 \mathrm{mg}$ /day); all but three were taking a regular inhaled corticosteroid (median dose $1000 \mu \mathrm{g} /$ day). Sixteen further patients were taking oral corticosteroids for other conditions.

MEASURES OF MORBIDITY

Use of short acting $\beta$ agonists

Four hundred and fifty five patients (13.6\%) had been prescribed 10 or more short acting $\beta$ agonist inhalers over the 12 months prior to the 


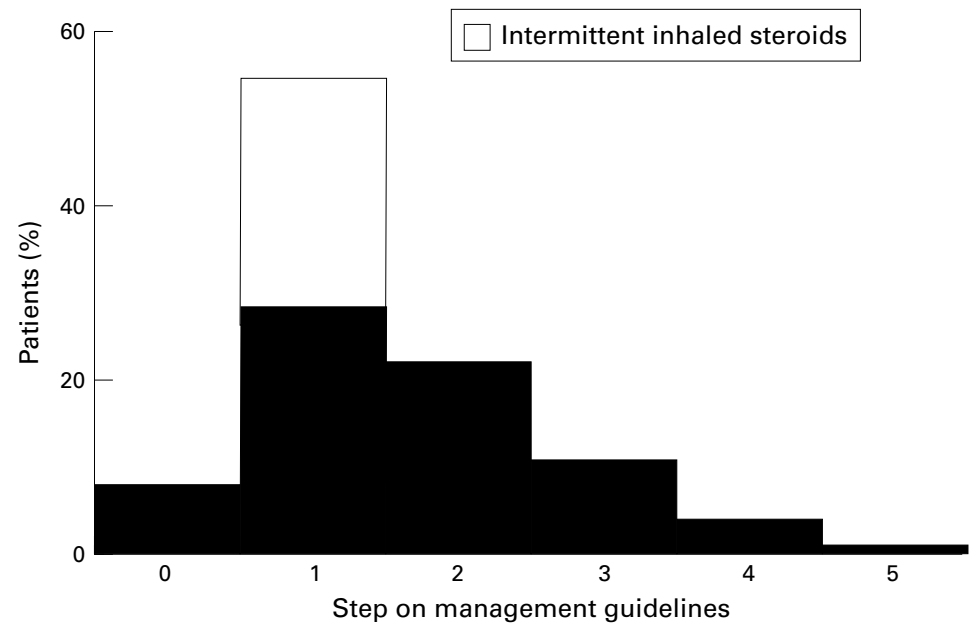

Figure 2 Percentage of the 3373 patients with asthma receiving treatment related to each step of the British guidelines for asthma management. The number of patients receiving no treatment and those on steps 1,2,3,4, and 5 were 268, 1834, 733, 359, 121, and 35, respectively. Step $1 b$ (clear box) describes patients who had been prescribed inhaled corticosteroids intermittently (fewer than 600 doses a year).

survey. Although patients on steps 4 and 5 of treatment were more likely to be taking such high doses, $54 \%$ of the patients were on steps 1 and 2 with 101 patients taking no regular inhaled corticosteroid (fig 3). One third of patients $(36 \%)$ were being prescribed short acting $\beta$ agonists for regular administration although in practice this group had had a median of two $\beta$ agonist inhalers in the last year (range 0-56 inhalers).

Courses of oral corticosteroids

In the previous 12 months 423 patients $(12.5 \%)$ not taking regular oral corticosteroids had required at least one course of prednisolone and $1.6 \%$ had required three or more courses. The pattern of use was similar to that for $\beta$ agonists in that patients on the higher steps of the asthma management guidelines were more likely to require oral corticosteroids (fig 4). Nevertheless, $60 \%$ of the patients were on steps 1 and 2 and $9 \%$ of patients having three or more courses were not taking a regular inhaled corticosteroid. There was a weak correlation between the use of $\beta$ agonist inhalers in the past year and the number of courses of oral corticosteroids prescribed (Spearman's rho $=0.26, \mathrm{p}<0.01)$.

Table 2 Asthma medication taken by the 3373 patients during the previous four years

\begin{tabular}{lr}
\hline Medication prescribed & No of patients \\
\hline Bronchodilator drugs & \\
Salbutamol & 2584 \\
Terbutaline & 458 \\
Salmeterol & 141 \\
Fenoterol + ipratropium & 10 \\
Ipratropium bromide & 62 \\
Theophylline & 57 \\
Nebulised salbutamol or terbutaline & 40 \\
Slow release oral $\beta$ agonist & 24 \\
Oxitropium bromide & 1 \\
Prophylactic medication & \\
Beclomethasone dipropionate & 1733 \\
Budesonide & 360 \\
Fluticasone propionate & 32 \\
Sodium cromoglycate & 47 \\
Nedocromil sodium & 1
\end{tabular}

*Numbers include all inhaled corticosteroids whether taken regularly (1219) or not (see Methods).
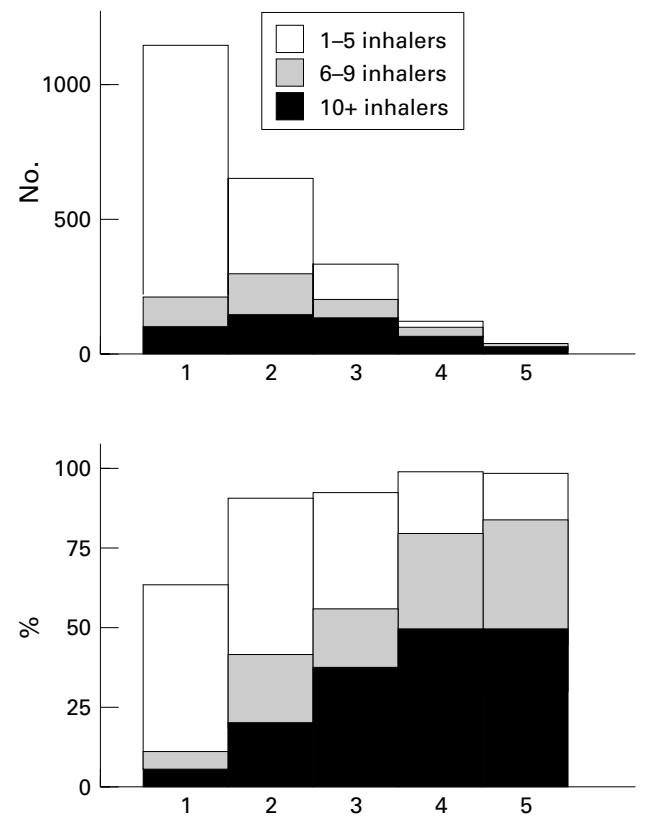

Step on asthma management guidelines

Figure 3 Number of patients requesting 1-5, 6-9 and $10+\beta$ agonist inhalers over the previous year in relation to the step on the asthma management guidelines. The data are expressed in absolute numbers and as a percentage of patients on each step of treatment.
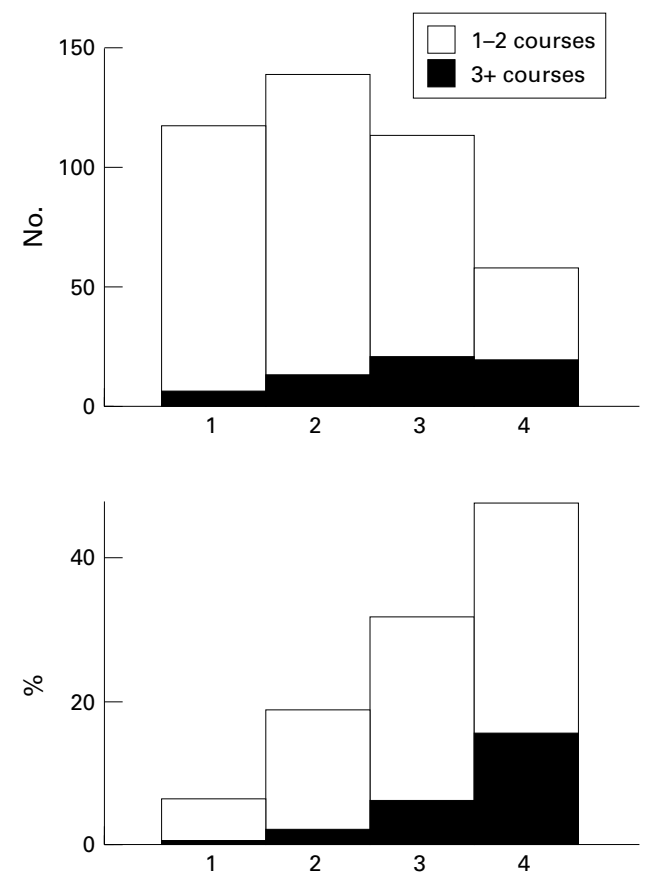

Step on asthma management guidelines

Figure 4 Number of patients requiring 1 or $2(n=369)$ or $3+(n=55)$ courses of oral prednisolone over the previous year in relation to their usual asthma therapy expressed as a percentage of patients on that step of treatment and in absolute numbers (total no of patients = 3322; patients on regular oral corticosteroid therapy (step 5) are excluded).

Effect of age and diagnosis of asthma and COPD Patients over the age of 50 were more likely to receive 10 or more $\beta$ agonist inhalers or a course of oral corticosteroids in the previous year but, since only $25 \%$ of patients were over 50 , removing these patients had only a small effect. Of the patients aged 5-50 years, $11.4 \%$ 
had received 10 or more $\beta$ agonist inhalers and $10.8 \%$ had received at least one course of oral steroids with $1.2 \%$ receiving three or more courses.

The patients with a diagnosis of asthma and COPD were older (mean age 69) than the patients with asthma alone, and they were more likely to have smoked in the past though the number of current smokers $(20 \%)$ was similar to the adult asthma group (18\%). The patients also appeared to have more severe disease with $12.8 \%$ on management step $5,18.3 \%$ on step $4,25 \%$ on step 3 , and only $25 \%$ on step 1 . Almost half of the patients $(47 \%)$ had received 10 or more short acting $\beta$ agonist inhalers and a third of those below step 5 had had a course of oral corticosteroids in the previous 12 months.

\section{Discussion}

This study describes the spectrum of asthma treatment in the community in relation to the 1995 British guidelines for asthma management. ${ }^{1}$ The diagnosis of asthma showed a marked age effect, falling from $17.3 \%$ of $10-14$ year old patients to less than $6 \%$ of adults over the age of 70 . The figures are similar to those obtained in recent surveys in Nottingham ${ }^{4}$ and elsewhere in the $\mathrm{UK}^{6-11}$ which, for diagnosed asthma, have been as high as $22.7 \%$ in schoolchildren and 5\% in adults.

Our study population appears to be representative of Nottinghamshire as judged by the similar age and sex distribution, Townsend scores, and prescribing patterns. Over $97 \%$ of patients in Nottinghamshire are registered with a general practitioner and $97 \%$ of practices are computerised. All the general practitioners made entries during the patient's visit and wrote prescriptions on a desk top computer so the data should cover all but an occasional acute prescription related to a home visit, hospital attendance, or possibly from a locum doctor. Since repeat prescriptions are usually generated from the computer by the receptionist and not included in the written record, it was not possible to carry out a formal validation exercise.

Of the $54 \%$ of patients on step 1, half had been prescribed an inhaled corticosteroid during the last four years but the dose was below our criterion for regular inhaled corticosteroid use (at least 600 puffs a year). This figure is arbitrary but is below the minimum recommended dose for any inhaled corticosteroid in the British National Formulary at the time of the study and, in fact, $88 \%$ of the patients had averaged less than 400 doses a year since commencing treatment. Slightly over one third of patients were taking an inhaled corticosteroid on a regular basis (step 2 or above), of whom one third were taking $800 \mu \mathrm{g} /$ day or more. The proportion of patients taking an inhaled steroid is similar to that reported in a recent questionnaire study ${ }^{12}$ and is higher than the figures from earlier studies using computerised general practice records from VAMP and AAH Meditel ${ }^{11}{ }^{13}$; none of the previous studies had data on the dose of inhaled corticosteroids used. Our study is therefore the first to be able to relate treatment of individuals in the community to steps on the asthma management guidelines.

We have no direct measure of how well asthma was controlled in our population but have used two indirect measures - the use of high doses of short acting inhaled $\beta$ agonists and the need for a course of oral corticosteroids. We have expressed both measures in two ways - as a percentage of the patients on each step of treatment and in absolute numbers. The first gives an indication of the extent to which patients at each step of treatment appear to be poorly controlled, whereas the second gives a measure of the morbidity in the community in relation to the regular treatment of the patients. The use of 10 or more short acting $\beta$ agonist inhalers as the cut off point is arbitrary but allows for some latitude for lost or spare inhalers.

The use of short acting $\beta$ agonist inhalers was high with $13.6 \%$ of patients prescribed 10 or more inhalers over the previous year. The use of high doses of $\beta$ agonists occurred more frequently in patients taking more prophylactic treatment, although more than half the patients requiring such high doses were on steps 1 and 2 of the treatment guidelines and almost a quarter were not taking a regular inhaled corticosteroid. Although not recommended by the guidelines, a third of patients were being prescribed a short acting inhaled $\beta$ agonist for regular administration. Patients did not necessarily adhere to this advice, however, since the median number of inhalers requested over the previous year was only two.

During the previous year $12.5 \%$ of patients not taking regular oral corticosteroids had had at least one course of prednisolone and $1.6 \%$ had required three or more courses. The need for a course of oral corticosteroids was closely related to asthma severity, as measured by the step of the asthma guidelines on which the patient was being treated, although more than half the patients requiring a course were on steps 1 and 2 .

Compliance with inhaled drugs for asthma is difficult to measure. We approached this by comparing the number of corticosteroid inhalers requested from the general practitioner with the number expected according to the dose prescribed. This measure has limitations since patients may lose their inhalers or obtain inhalers from other sources, they may waste doses by actuating to air before use, and inhalers usually contain around $10 \%$ more than the stated number of doses. Nevertheless, $39 \%$ of patients on regular inhaled corticosteroids had requested less than $80 \%$ of the expected dose which suggests some problems with compliance or perhaps a dose reduction by the patient once their asthma was well controlled.

Although our data are descriptive, certain conclusions can be drawn. Both measures of morbidity - the request for more than $10 \beta$ agonist inhalers a year and the need for a course of oral corticosteroids-occurred fairly frequently and both were more likely to occur in patients already taking high doses of prophylactic medication. However, since most 
patients in the community are on steps 1 and 2 of the treatment guidelines, more than half the patients requiring high doses of $\beta$ agonists or a course of oral corticosteroids are on steps 1 and 2. Assuming our data to be representative for the UK as a whole, the number of patients on steps 1 or 2 of the asthma guidelines requiring 10 or more $\beta$ agonist inhalers or a course of oral corticosteroids in the last year would be 350000 and 360000 , respectively. These patients may benefit from an increase in prophylactic treatment. The data also highlight a sizeable group of patients who appear to have inadequate control despite taking high doses of prophylactic medication. Further work is needed to determine the extent to which compliance is a problem in these patients or whether they have persisting symptomatic asthma despite treatment which would generally be considered as appropriate. Use of high doses of $\beta$ agonists and the need for a course of oral corticosteroids are indices that could be used as measures of asthma control on a community basis in future studies.

We thank Steven Hough at the North Nottinghamshire FHSA and Tim Harrison for comments on the manuscript. We are especially grateful to the following practices and particularly the practice managers and computer staff for help with the study: Dr A Hutton and partners in Collingham, Newark; Dr J Bilkhu and partners, Radcliffe on Trent; and the following practices in and partners, Radcliffe on Trent; and the following practices in Dr F Coutts and partners, Arnold; and Dr P D Sprackling and partners, Lenton.
Dr L Walsh is supported by a National Asthma Campaign project grant and the study was supported by a donation from Astra Draco.

1 British Thoracic Society, et al. The British guidelines on asthma management: 1995 review and position statement. Thorax 1997;52 (Suppl 1):S1-21.

2 Walsh LJ, Wong CA, Pringle $M$, et al. Use of oral corticosteroids in the community and the prevention of secondary osteoporosis: a cross sectional study. BMF 1996; 313:344-6.

3 Wilson S. Aiming for health in the year 2000: the annual report of the Director of Public Health 1995. Nottingham Health District, 1995.

4 Venn A, Lewis S, Cooper M, et al. Self-reported prevalence of asthma and wheeze in Nottinghamshire secondary schoolchildren. Am f Respir Crit Care Med 1997;155:A75.

5 Venn A, Lewis S, Cooper M, et al. Recent changes in the prevalence of asthma and wheezing illness in Nottinghamshire primary school children. Am f Respir Crit Care Med 1997;155:A75.

6 Kaur B, Anderson RH, Austin J, et al. Prevalence of asthma symptoms, diagnosis, and treatment in 12-14 year old children across Great Britain (international study of asthma and allergies in childhood, ISAAC, UK). BMF 1998;316: 118-24.

7 Omran M, Russell G. Continuing increase in respiratory symptoms and atopy in Aberdeen schoolchildren. BMF 1996;312:34.

8 Rona RJ, Chinn S, Burney PGJ. Trends in the prevalence of asthma in Scottish and English primary school children 1982-92. Thorax 1995;50:992-3.

9 Ninan TK, Russell G. Respiratory symptoms and atopy in Aberdeen schoolchildren: evidence from two surveys 25 years apart. BMF 1992;304:873-5.

10 Renwick DS, Connolly MJ. Prevalence and treatment of chronic airways obstruction in adults over the age of 45 . Thorax 1996;51:164-8.

11 Roberts SJ, Bateman DN. Which patients are prescribed inhaled anti-asthma drugs? Thorax 1994;49:1090-5.

12 Marks GB, Burney PGJ, Premaratne UN, et al. Asthma in Greenwich, UK: impact of the disease and current management practices. Eur Respir f 1997;10:1224-9.

13 Warner JO. Review of prescribed treatment for children with asthma in 1990. BMF 1995;311:663-6. 